\title{
Assessing the 'cyclodiode G-probe' using a grey scale test: reproducibility and differences between probes
}

\begin{abstract}
Aims

1. To determine the number of clinicians performing cyclodiode therapy who reuse the 'G-probe' used for the delivery of cyclodiode therapy.

2. To show a simple method to assess the output of the 'G-probe' that can be used in the clinical setting.
\end{abstract}

Methods A total of 71 questionnaires were sent to ophthalmologists who have an Oculight SLx Iris Medical Diode Laser. Ophthalmologists were asked as to whether they performed cycloablative therapy using the 'G-probe' and whether they reused the G-probe. They were also asked as to the frequency of any reuse of probes.

To determine the output of the 'G-probe', paper copies of a custom-made grey scale chart containing graded blocks of increasing shades of grey densities were produced. A special probe holder was made so that the G-probe tip could be held at a fixed distance from the grey scale chart. Laser burns were made on the grey scale using this arrangement and measurements of the burn size were made. After using 'standard settings' of $2000 \mathrm{~ms}$ and $2000 \mathrm{~mW}$, 'threshold' burns were defined. Five new probes (with two different operators) were tested to assess the interprobe, interoperator, and intersheet variability of test. Probes were then tested for the burn size produced between 1000 and $3000 \mathrm{~mW}$, and 1000 and $3000 \mathrm{~ms}$.

Results Results from the questionnaire showed that of the 44 respondents $(62.0 \%$ response), $93.2 \%$ performed cyclodiode therapy with 58.5\% reusing the G-probe. Among them, $56.1 \%$ reused probes on more than one occasion.

P Hossain 1 , G Ghosh² and SA Vernon ${ }^{2}$

Results from testing a new G-probe on the grey scale chart showed that with 'standard settings', highly reproducible burns at grey density 8 could be produced. No significant interprobe, interoperator, and intersheet variations were noted. Above $3 \mathrm{~J}$ of laser energy, the test could detect a $20 \%$ increase in energy settings and it was found that at levels of $4 \mathrm{~J}$ or above, alterations to the power setting had a greater influence on burn production than alterations to the time setting.

Conclusions This study demonstrates

1. that many clinicians in the UK reuse G-probes,

2. a simple, quick, and highly reproducible method to assess the laser output from the G-probe used for cyclodiode therapy.

The method can help the ophthalmic surgeon to test the G-probe prior to commencement of therapy and with a standard treatment protocol, may produce a more predictable intraocular pressure reduction.

Eye (2003) 17, 167-176. doi:10.1038/

sj.eye. 6700285

Keywords: glaucoma; anterior segment; ciliary epithelium; laser; intraocular pressure

\section{Introduction}

Cyclophotocoagulation is an effective form of therapy to reduce intraocular pressure. ${ }^{1-3}$ This pressure lowering effect is achieved by thermal ablation of the ciliary body, ${ }^{4,5}$ causing reduced aqueous production. Using a standardised treatment protocol and a diode laser, cyclophotocoagulation (cyclodiode therapy)
${ }^{1}$ Academic Department of Ophthalmology University of Nottingham Eye, Ear, Nose \& Throat Centre Queen's Medical Centre Nottingham, UK

${ }^{2}$ Department of Ophthalmology Eye, Ear, Nose \& Throat Centre Queen's Medical Centre Nottingham, UK

Correspondence: Dr SA Vernon

Eye, Ear, Nose \&

Throat Centre

Queen's Medical Centre Nottingham NG7 2UH, UK Tel: + 441159249924

Fax: +44 1159709963

E-mail: parwez.hossain@ nottingham.ac.uk

Received: 22 October 2001 Accepted in revised form: 15 May 2002 
produces intraocular pressure reduction with relatively few complications. ${ }^{6}$

Many reports on this therapy utilise a special probe called the 'G-probe' (Iris Medical Instruments Inc., Mountain View, CA, USA) and an infrared diode laser $(810 \mathrm{~nm})$ (Oculight SLx, Iris Medical Instruments Inc., Mountain View, CA, USA). ${ }^{6-9}$ It is recommended that each probe should only be used once for one session of laser treatment. ${ }^{10}$ However, many surgeons reuse individual G-probes (PA Bloom, 1999, personal communication), despite knowing the risks associated with reusing a single-use device. ${ }^{11}$ To what extent surgeons reuse the probes is not known. It is likely that with reuse, there is a gradual reduction of laser output of the G-probe resulting in reduced effectiveness of ciliary body ablation. ${ }^{10}$ However, it is technically difficult to measure the direct laser output of the G-probe in a clinical setting. ${ }^{13,14}$

We assessed the proportion of clinicians reusing the Gprobe after sending a questionnaire to ophthalmologists to whom an Iris Medical Diode Laser was supplied. In a separate study, we developed a new method to assess the output from the G-probe using a custom-made grey scale chart. The method was found to be quick, simple to perform, and highly reproducible, providing information on the output of old and new probes.

\section{Materials and methods}

Three separate studies were conducted and are described under the following categories:

1

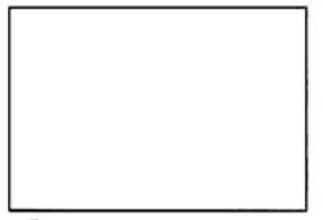

5

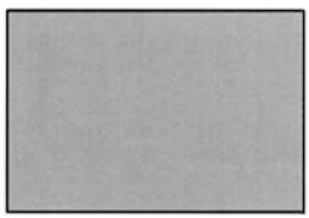

9

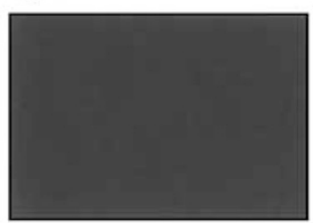

2

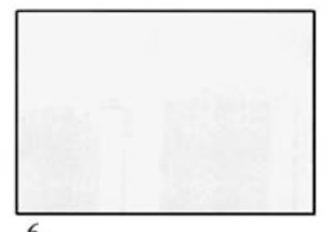

6

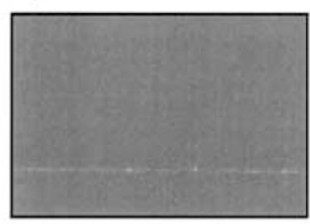

10

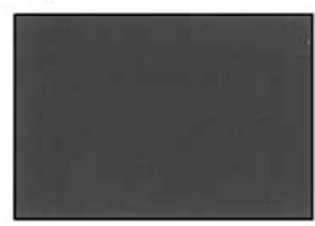

(A) A questionnaire-based survey of ophthalmologists aimed to investigate the current use of the G-probe.

(B) A pilot study aimed to assess a method to test the output of the G-probe using a custom-made grey scale chart.

(C) Using the developed grey scale chart test, a study was performed to assess the effect of different laser settings to produce laser burns on the grey scale chart test.

\section{Study A: questionnaire-based survey of ophthalmologists}

A total of 71 ophthalmologists in the UK, who have an Oculight SLx Iris Medical Laser unit (ownership details were provided by Iris Medical Instruments), were sent a single-page questionnaire. The clinicians were asked as to whether they use the G-probe in conjunction with the Iris Medical Laser to perform cycloablation on patients. Further questions were asked on the reuse of the probes and the frequency of reuse between different treatment sessions.

\section{Study B: a pilot study to develop a 'grey scale test'}

Design of grey scale chart A grey scale chart, containing a palette of different grey densities, was designed using Power Point software (Microsoft Office 97, Microsoft Inc.). Initially, all grey shades were selected (15 shades). Several copies of the chart were printed on to white paper (paper density $80 \mathrm{~g} / \mathrm{m}$ ), using an inkjet printer (Hewlett-Packard 720c, San Diego, USA) (Figure 1). Each

3

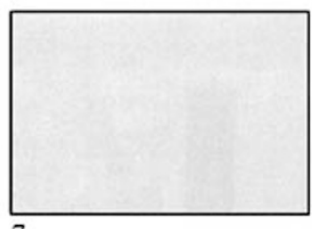

7

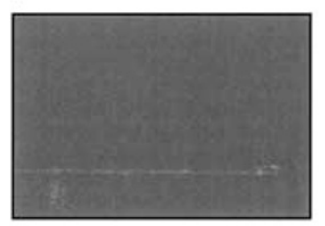

11

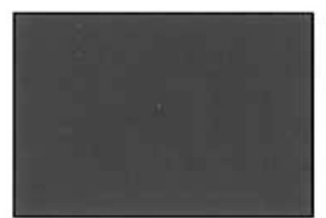

4
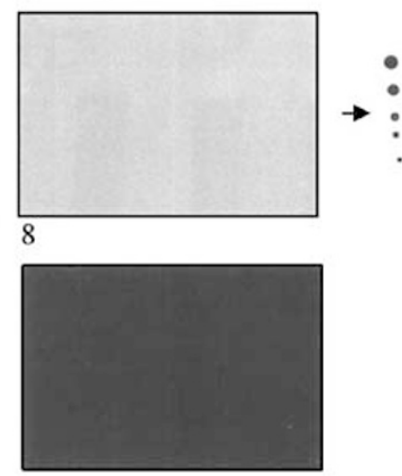

12

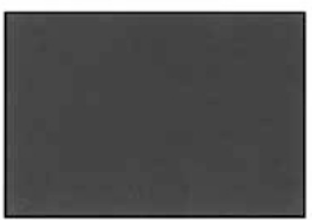

Figure 1 Custom-made grey scale chart used in the study; the above image shows that the chart contains a palette of grey shade blocks of increasing grey densities. 
shade of grey on the palette was graded for grey density by comparing it to a standard grey scale (Kodak Control Grey Scale 14, Eastman Kodak Company, Rochester, NY, USA). Some consecutive grey shades showed similar densities to each other and when this occurred, one of the shades was excluded. A final number of differing 12 grey shades was found. All comparisons were performed by three independent observers who assigned a number to each grey shade. The value of each assigned number corresponded to the Kodak Control Grey Scale and ranged from 1 to 12 with increasing order of grey density.

Design of probe holder A probe holder was produced from black plastic by the Mechanical Engineering Department, Nottingham University (Figure 2a) (available from Mr S Vernon, Department of Ophthalmology, Queens Medical Centre, Nottingham, UK). An inner aperture in the holder permitted the Gprobe to be held vertical to the plane of the holder. The design ensured that when the leading edge of the probe reached the lower flat surface of the holder, the distance between the probe tip and the grey scale was constant and was kept at a distance of $2 \mathrm{~mm}$. This arrangement is shown in Figure 2b.

Producing a 'test' burn To produce a burn the following protocol was followed: The tip of the G-probe was cleaned with isopropyl alcohol swab (Sterets, Seaton Healthcare Group, Oldham, UK) and allowed to dry in air. Holding the G-probe vertically, the probe was inserted into the inner aperture of the holder so that the leading edge of the probe reached the lower flat surface of the holder. The whole arrangement of the probe and holder was placed over the grey scale sheet so that the probe position coincided over a selected grey shade. With both the holder and G-probe held firmly over the grey scale sheet, the laser was then fired in an attempt to produce a burn on the sheet.

Determining 'threshold burns' at 'standard settings' A new G-probe was used in the pilot study. The diode laser was set at the 'standard settings' for energy exposure used in our unit for cyclodiode treatment $(2000 \mathrm{~mW}, 2000 \mathrm{~ms}){ }^{6}$ Three burns were attempted on each grey shade on the
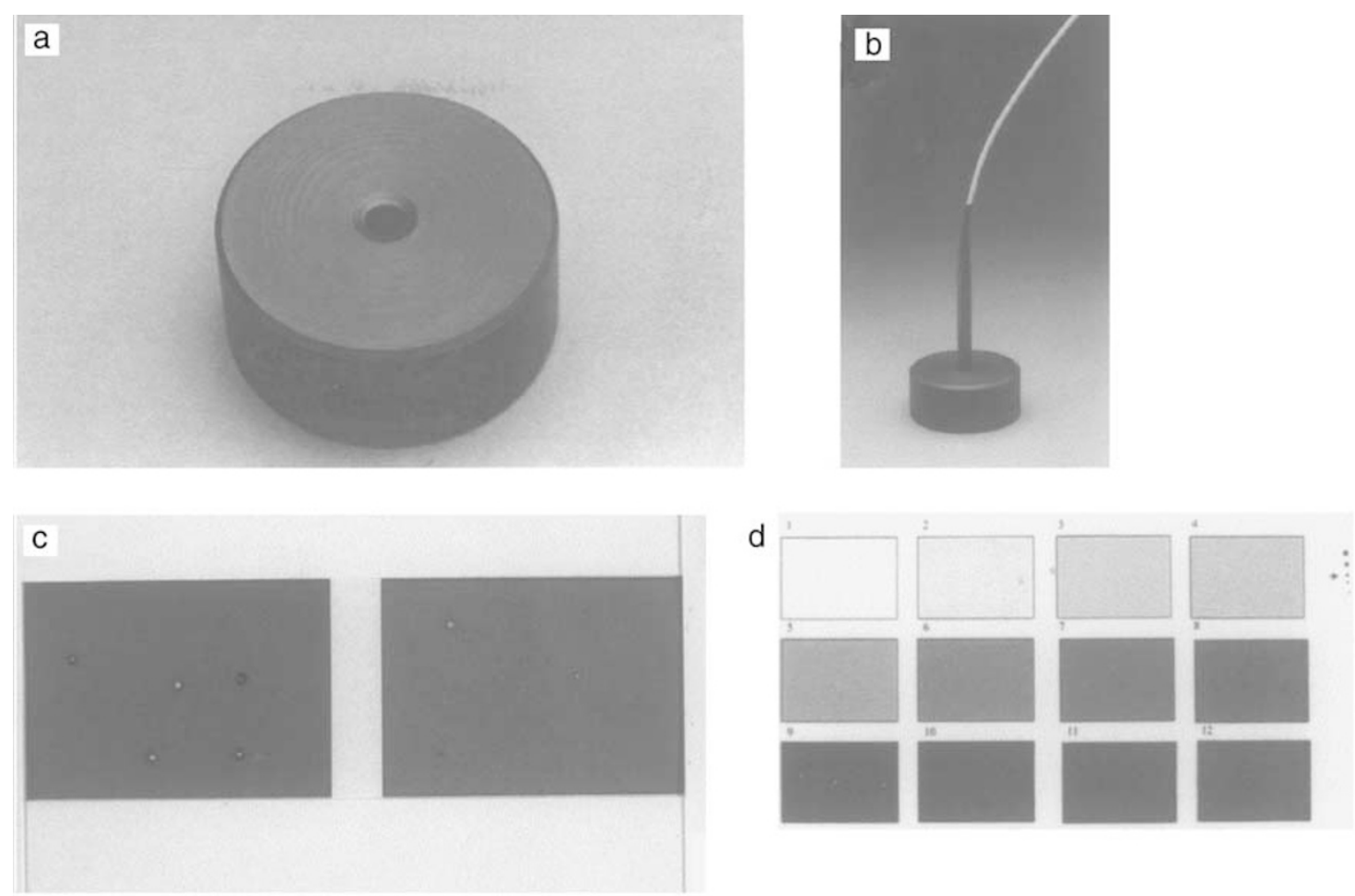

Figure 2 (a) Image of the probe holder. (b) Image of the arrangement of the 'G'-probe in the probe holder. (c) Image of two adjacent grey shades (left block, grey shade 7; right block, grey shade 8) with threshold and nonthreshold burns. The left block shows five attempted burns, four of which produce visible discoloration and one burn fails to make any mark, all areas of discoloration are less than $1500 \mu \mathrm{m}$ in size. On the right block, all five burns produced visible discoloration of $1500 \mu \mathrm{m}$ or more. (d) Photograph of grey scale showing a scale of different burn sizes (right-hand corner, $1700 \mu \mathrm{m}$ size is indicated by black arrow) to allow the treating clinician to compare the test burns with the size of burn produced by the 'standard settings' of $2 \mathrm{~W} / 2 \mathrm{~s}$. A series of such burns has been produced on grey shades 8 and 9 . 
grey scale chart. The test was repeated on different charts (printed on different batches of paper) to check for interchart variations. The test was also repeated by different operators to check for inter- and intraoperator variations; after each shot, the probe tip was cleaned with isopropyl alcohol swab.

The exposed charts were examined under a dissecting microscope (Nikon, Kingston-upon-Thames, UK). Burns were identified by any discoloration at the site of laser application (Figure 2c), and using a microscope graticule (Graticules, Hamblin, UK) the diameter of each burn was measured. Measurements of the three attempted burns for each grey shade were averaged and expressed as the 'burn size' in micrometres \pm standard deviation $(\mu \mathrm{m} \pm \mathrm{SD})$.

After this initial assessment, a further five new probes were tested on different sheets and by different operators to assess the inter-intra-probe, intersheet and intra-interoperator variations of the technique. The five probes were then used to determine the laser power setting that produced 'threshold' burns. This was done by testing each probe on a modified sheet containing only blocks of grey shade 8 . Then each probe was tested using laser power output settings from 1000 to $2100 \mathrm{~mW}$, increasing by $100 \mathrm{~mW}$ steps. Throughout this assessment the time setting was kept constant at $2000 \mathrm{~ms}$ and after each shot, the probe tip was cleaned with isopropyl alcohol. After completing these assessments, the sheets were analysed for the burns produced as described earlier.

\section{Study C: using the 'grey scale test' to assess the output of the G-probe using different laser settings}

Assessing changing laser output parameters The effect of changing output parameters was then assessed. Using the burn protocol in the pilot study, three separate burns were attempted on each grey shade on the grey scale palette. Measurements of the burn size and burn score were made. The burning effect of the G-probe was assessed at power settings of 1000, 1500, 2000, 2500, and $3000 \mathrm{~mW}$, while the exposure time was kept constant at $2000 \mathrm{~ms}$. The burn effect of the G-probe was then assessed at different time settings of 1000, 1500, 2000,
2500, and $3000 \mathrm{~ms}$, while the power output was kept constant at $2000 \mathrm{~mW}$. The grey scale sheets were then analysed for the burns produced, as described earlier, and the results for each time/power setting where the total energy value was similar were compared (eg 1000 $\mathrm{mW} / 2000 \mathrm{~ms}$ setting was compared to the $1000 \mathrm{~ms}$ / $2000 \mathrm{~mW}$ setting, ie total energy value $2 \mathrm{~J}$ ).

Comparison of laser burns produced by new and old probes A new G-probe was assessed using the protocol given in the pilot study (under the heading 'determining 'threshold burns' at 'standard settings', ie $2000 \mathrm{~ms} /$ $2000 \mathrm{~mW}$ ). The laser burns produced were compared to an old probe, which had been used in six previous sessions of cyclodiode therapy.

Statistics The results from the five new probes were analysed using statistical tests. Burn scores and burn sizes were assessed using the Mann-Whitney rank test. A one-way ANOVA was used to compare the burn sizes recorded on grey shades 8 and 9. Data from the time and power variation part of the study were analysed by a paired $t$-test, a $P$-value of $<0.05$ was taken as statistically significant.

\section{Results}

Results of Study A: questionnaire-based survey of ophthalmologists

Of the 71 ophthalmologists to whom a questionnaire was sent, 44 replied (62\% response rate). Of the respondents, $88.6 \%$ (41 ophthalmologists) used the G-probe to perform cycloablative therapy and $58.5 \%$ of these clinicians reused the probe. Tables 1 and 2 summarise the results of the questionnaire.

\section{Results of Study B: a pilot study to develop a 'grey scale test'}

'Burn score', 'threshold burn' and 'threshold' Following the pilot study, it was found that using 'standard settings'

Table 1 Summary of the responses to the questionnaire

\begin{tabular}{ll}
\hline Question & Number of responses (\% in brackets) \\
\hline Number of clinicians performing cyclodiode therapy & $41(93.2 \%$ of respondents) \\
Number of clinicians using the G-probe & $39(95.1 \%$ of clinicians performing cyclodiode therapy) \\
Number of clinicians reusing probes & $24(58.5 \%$ of clinicians performing cyclodiode therapy) \\
Number of clinicians not reusing probes & $15(36.6 \%$ of clinicians performing cyclodiode therapy) \\
Number of clinicians who do not know & $1(2.4 \%$ of clinicians performing cyclodiode therapy) \\
\hline
\end{tabular}


Table 2 Number of responses for different levels of reuse (and of no reuse) of probes by responding clinicians with the frequency of cycloablative treatments performed in their practice

\begin{tabular}{|c|c|c|c|c|c|}
\hline \multirow[t]{2}{*}{ Number of probe reuses before disposal } & \multicolumn{5}{|c|}{ Number of cycloablative treatments performed } \\
\hline & Once per year & Once per 6 months & Once per 3 months & Once per month & 1-5 times per week \\
\hline No reuse & 1 & & 4 & 5 & 6 \\
\hline 1 reuse & & & 1 & & \\
\hline $2-5$ reuses & & & 2 & 3 & 6 \\
\hline $6-10$ reuses & & & & 1 & 4 \\
\hline $11-15$ reuses & & 1 & & & \\
\hline $16-20$ reuses & & & 2 & & 1 \\
\hline$>20$ reuses & & & 1 & & 2 \\
\hline
\end{tabular}

(2000 ms and $2000 \mathrm{~mW}$ ), three consistent burns (burn size greater than $1500 \mu \mathrm{m}$ ) could be produced on grey shade 8 (Figure 2d). At lighter grey shades, consistent burns could not be produced using these 'standard settings'. From this initial study, we defined the terms 'threshold burn' and 'threshold grey shade'. A 'threshold burn' was defined as a visible burn in excess of $1500 \mu \mathrm{m}$ in size and, when all three laser shots produced 'threshold burns' on a particular grey shade, then this grey shade value was taken as the 'threshold grey shade'.

Reproducibility and interoperator variability of the method Using five new probes at 'standard settings', no significant difference was found in the burn size when each of the five new probes (Figure 3a) was tested on grey shade 8 . When burns were produced on grey shade 9 , burn sizes were found to be significantly larger on grey shade 9 than on grey shade $8(P<0.001$, one-way ANOVA) (Figure 3a). Using five different grey scale sheets (printed on different batches of paper) and two different operators, threshold burns were produced consistently on grey shade 8 . No interoperator, intraoperator, or intersheet differences were found (Figure 3b).

\section{Minimum power setting to produce a 'threshold}

burn' Figure 4a shows the burn sizes produced by the five new probes (on grey scale 8), with power settings ranging from 1000 to $2100 \mathrm{~mW}$ but keeping the laser time exposure constant at $2000 \mathrm{~ms}$. Above $1800 \mathrm{~mW}$, all the probes produced consistent 'threshold burns'; however, below $1700 \mathrm{~mW}$ the burns produced were less consistent.

Figure 4a also shows that the testing system could detect statistically significant changes $(P<0.05)$ in burn diameter when the laser power setting was increased by $20 \%$ or more above $1500 \mathrm{~mW}$.
Results of Study C: using the 'grey scale test' to assess the output of the G-probe using different laser settings

Assessing changing laser output parameters Figure $4 \mathrm{~b}$ shows the effect of changing exposure time while keeping the laser power setting at $2000 \mathrm{~mW}$. The graph shows that the threshold burns on grey shade 8 can be produced on grey shade 7 if the laser exposure time is increased to $3000 \mathrm{~ms}$. Figure $4 \mathrm{~b}$ also shows that increasing the laser exposure time also increases the diameter of the burns produced.

Figure 4c shows the effect of different laser power settings while keeping the laser exposure time constant at $2000 \mathrm{~ms}$. Threshold burns can be achieved at lighter grey shades if the power setting is set above $2500 \mathrm{~mW}$, and a power setting of $3000 \mathrm{~mW}$ can produce threshold burns at grey shade 5 . Figure $4 \mathrm{c}$ also shows that larger burn diameters are produced with higher laser powers.

Comparing the effect of changing power settings with time settings for a given laser output Using a paired $t$-test, the effects of changing power and time settings for a given total energy output were compared (Table $3 a$ and $b$ ), and the burn sizes for each energy level are compared with differing time and power settings, in Figure 5a. When total energy levels were greater than $4 \mathrm{~J}$, altering the power setting had a greater effect in producing larger burns than by increasing the time setting, even though the overall energy output was the same.

Assessing the output of a 'new' probe with an 'old' probe $\mathrm{A}$ new G-probe produced nonthreshold burns at grey shade 7 and produced threshold burns on grey shade 8 . Using the old probe, threshold burns were only produced on grey density 9 (Figure $5 b$ ). The new probe also produced larger burns compared to an old probe at any given grey shade (Figure $5 b$ ). 

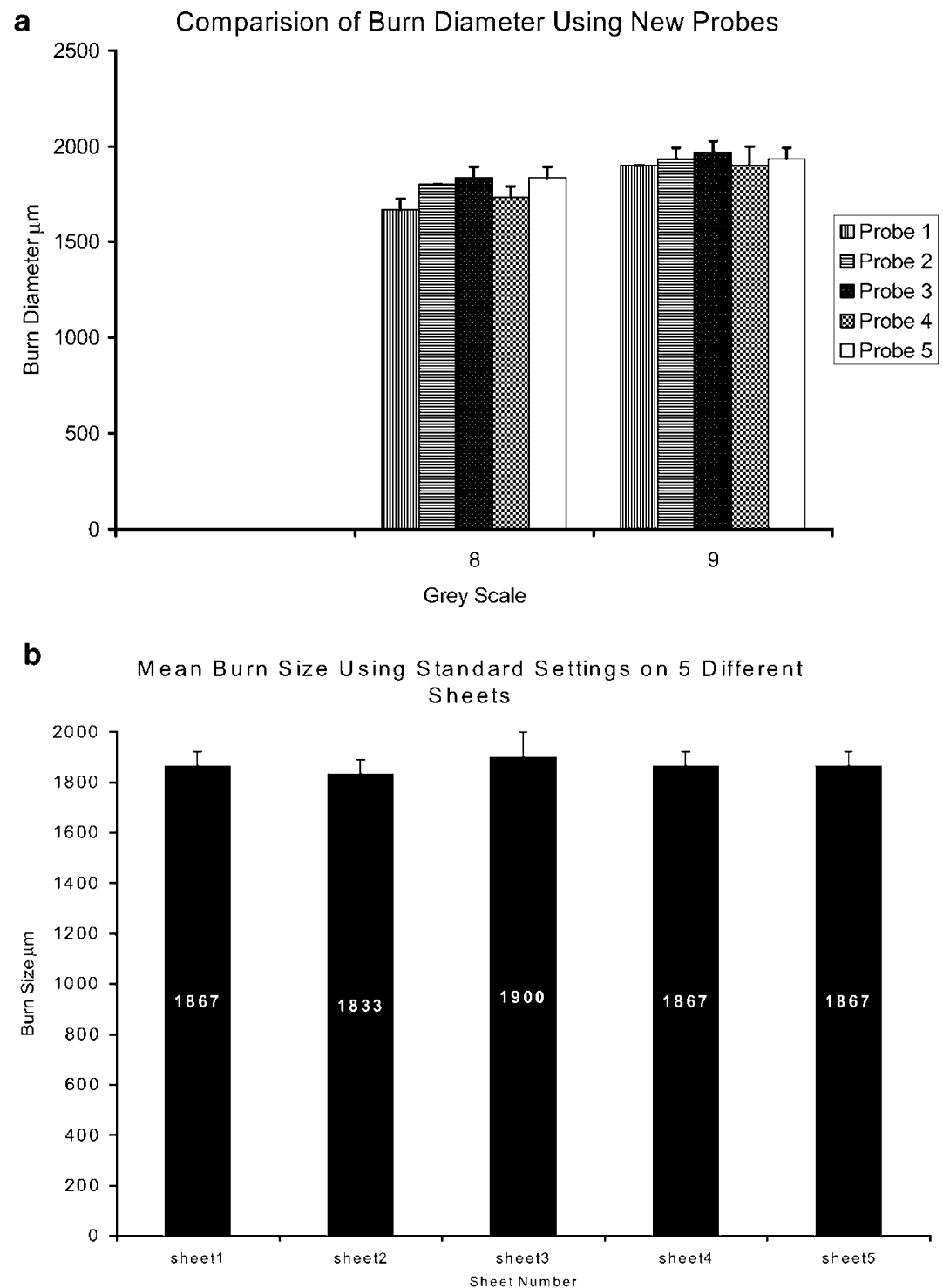

Figure 3 (a) Mean burn diameters $(\mu \mathrm{m}+\mathrm{SD}$ ) produced by five new probes on grey scale 7-9, using 'standard settings' of $2000 \mathrm{~ms}$ and $2000 \mathrm{~mW}$. (b) Graph showing the mean burn diameters $(\mu \mathrm{m}+\mathrm{SD})$ produced on grey scale 8 on five different sheets by one operator using 'standard settings' of $2000 \mathrm{~ms}$ and $2000 \mathrm{~mW}$.

\section{Discussion}

Cyclodiode therapy using the G-probe and diode laser is proving to be a popular and effective form of therapy to treat intractable high intraocular pressure..$^{2,3-9,14,15}$ It is recommended that the G-probe is used for a single treatment session and reuse is not advised. ${ }^{11}$ However, it is clear from this study that many clinicians in the UK reuse probes. This study found that those clinicians who performed cyclodiode therapy on a weekly basis were reusing probe the most (69\% of clinician performing weekly cyclodiode therapy reuse probes). It is likely that probes become less effective with reuse, resulting in reduced efficacy of the laser treatment; thus, for clinicians who reuse probes, it is important that there is a method to assess the output of the G-probe before use.

The method advised by the manufacturers of Oculight SLx Diode Laser (Iris Medical Instruments Inc., Mountain View, CA, USA) to measure the output of $\mathrm{G}$-probe is to use a calibrated laser power meter. The technique involves immersing the G-probe tip in a glass Petri dish filled with deionised water (to prevent the scattering of light at the probe tip). The calibrated laser power meter is then positioned underneath the dish so that emitted laser light can be measured. Calculations are then required to compensate for expected losses as a 

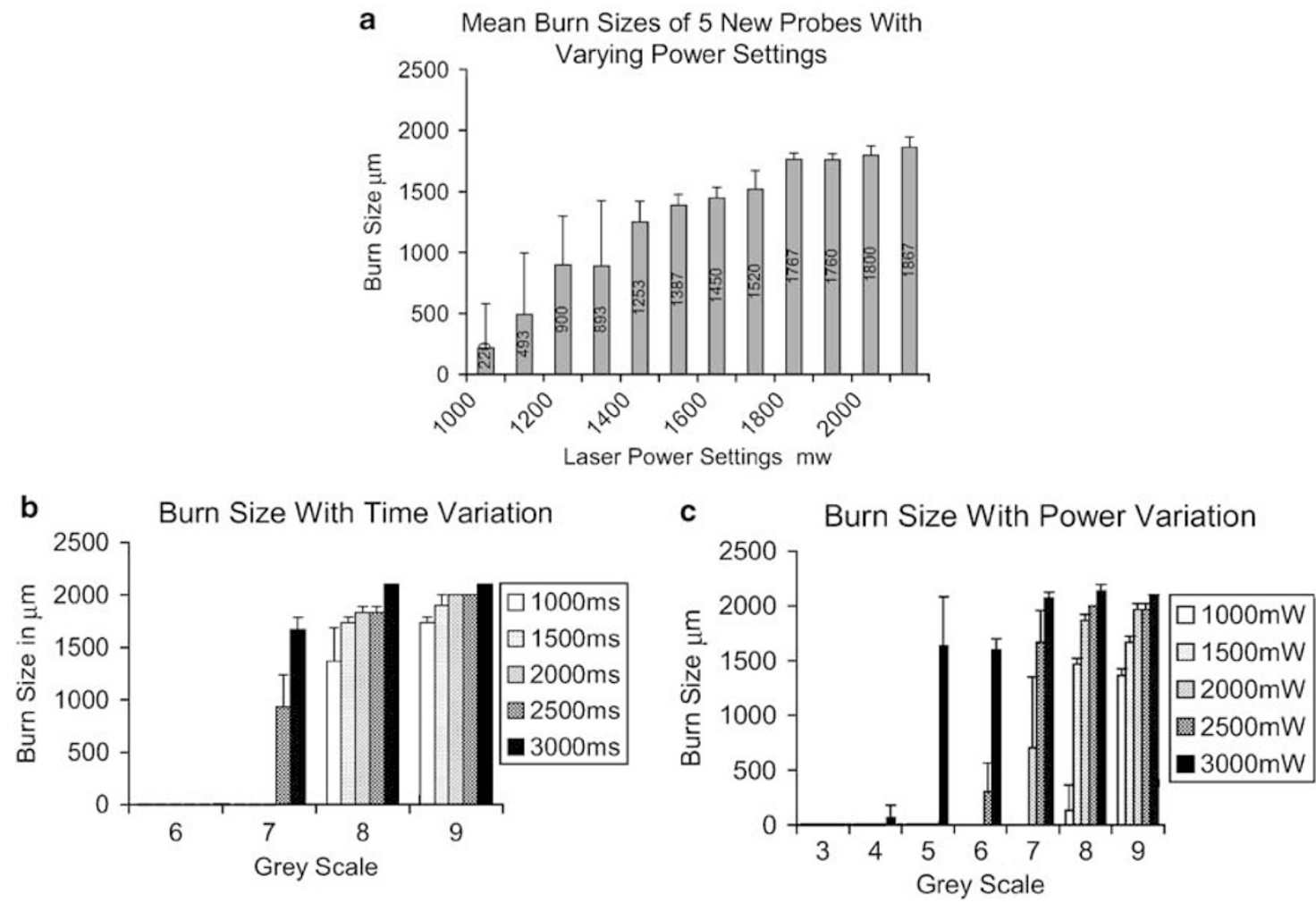

Figure 4 (a) Mean burn sizes produced $(\mu \mathrm{m}+\mathrm{SD}$ ) by five new probes when the power setting was varied from 1000 to $2100 \mathrm{~mW}$. The test was performed on grey scale 8 keeping the time setting constant at $2000 \mathrm{~ms}$. (b) Mean burn size produced $(\mu \mathrm{m}+\mathrm{SD}) \mathrm{on}$ grey scale 6-9 using different time settings. The power setting was kept constant at $2000 \mathrm{~mW}$. (c) Mean burn size produced ( $\mu \mathrm{m}+\mathrm{SD})$ on grey scale 3-9 using different power settings. The power setting was kept constant at $2000 \mathrm{~ms}$.

Table 3

\begin{tabular}{|c|c|c|c|c|c|c|c|}
\hline & \multicolumn{7}{|c|}{ Grey scale } \\
\hline & 3 & 4 & 5 & 6 & 7 & 8 & 9 \\
\hline \multicolumn{8}{|c|}{ (A) Mean burn size ( $\mu m$ ) produced for each time and power setting for each grey scale } \\
\hline $1000 \mathrm{~mW} / 2000 \mathrm{~ms}$ & 0 & 0 & 0 & 0 & 0 & 133 & 1367 \\
\hline $1500 \mathrm{~mW} / 2000 \mathrm{~ms}$ & 0 & 0 & 0 & 0 & 0 & 1467 & 1667 \\
\hline $2000 \mathrm{~mW} / 2000 \mathrm{~ms}$ & 0 & 0 & 0 & 0 & 700 & 1867 & 1967 \\
\hline $2500 \mathrm{~mW} / 2000 \mathrm{~ms}$ & 0 & 0 & 0 & 300 & 1667 & 2000 & 1967 \\
\hline $3000 \mathrm{~mW} / 2000 \mathrm{~ms}$ & 0 & 67 & 1633 & 1600 & 2067 & 2133 & 2100 \\
\hline $2000 \mathrm{~mW} / 1000 \mathrm{~ms}$ & 0 & 0 & 0 & 0 & 0 & 1367 & 1733 \\
\hline $2000 \mathrm{~mW} / 1500 \mathrm{~ms}$ & 0 & 0 & 0 & 0 & 0 & 1733 & 1900 \\
\hline $2000 \mathrm{~mW} / 2000 \mathrm{~ms}$ & 0 & 0 & 0 & 0 & 0 & 1833 & 2000 \\
\hline $2000 \mathrm{~mW} / 2500 \mathrm{~ms}$ & 0 & 0 & 0 & 0 & 933 & 1833 & 2000 \\
\hline $2000 \mathrm{~mW} / 3000 \mathrm{~ms}$ & 0 & 0 & 0 & 0 & 1667 & 2100 & 2100 \\
\hline \multicolumn{8}{|c|}{ (B) Calculated P-values (paired t-test) comparing equivalent energy settings, for example, $1000 \mathrm{~mW} / 2000 \mathrm{~ms}$ vs $2000 \mathrm{~mW} / 1000 \mathrm{~ms}$} \\
\hline $1000 \mathrm{~mW} / 2000 \mathrm{~ms}$ vs $1000 \mathrm{~ms} / 2000 \mathrm{~mW}$ & NA & NA & NA & NA & NA & 0.06 & 0.03 \\
\hline $1500 \mathrm{~mW} / 2000 \mathrm{~ms}$ vs $1500 \mathrm{~ms} / 2000 \mathrm{~mW}$ & NA & NA & NA & NA & NA & 0.02 & 0.27 \\
\hline $2000 \mathrm{mw} / 2000 \mathrm{~ms}$ vs $2000 \mathrm{~ms} / 2000 \mathrm{~mW}$ & NA & NA & NA & NA & 0.21 & 0.42 & 0.42 \\
\hline $2500 \mathrm{~mW} / 2000 \mathrm{~ms}$ vs $2500 \mathrm{~ms} / 2000 \mathrm{~mW}$ & NA & NA & NA & 0.19 & 0.03 & 0.04 & 0.42 \\
\hline $3000 \mathrm{~mW} / 2000 \mathrm{~ms}$ vs $3000 \mathrm{~ms} / 2000 \mathrm{~mW}$ & NA & 0.42 & 0.02 & $<0.01$ & 0.02 & 0.42 & NA \\
\hline
\end{tabular}

A $P$-value $<0.05$ was taken as statistically significant. NA, data not calculable. 


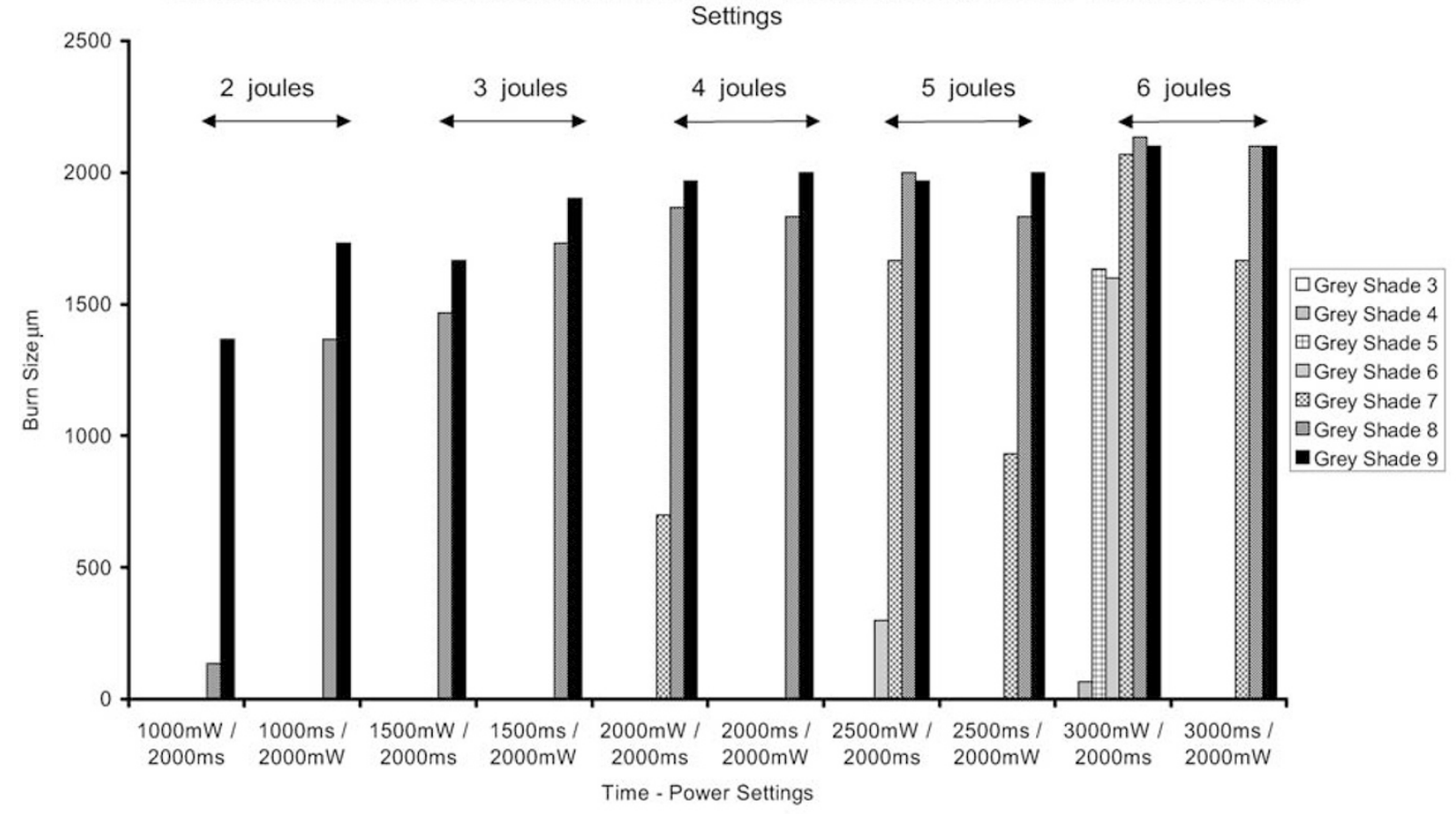

b

Comparison of Burns Produced at

'Standard Settings' For a New and Old

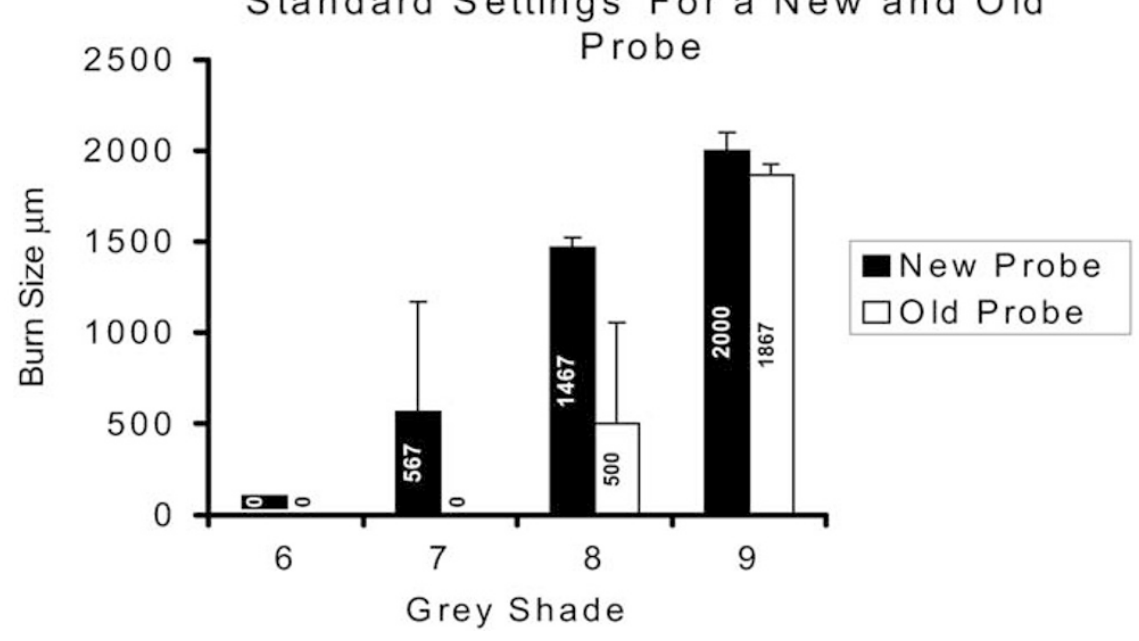

Figure 5 (a) Comparison of mean burn sizes $(\mu \mathrm{m}+\mathrm{SD})$ produced by alteration of time and power settings at equivalent energy settings for different grey shades (grey shades 3-9). (b) Mean burn size $(\mu \mathrm{m}+\mathrm{SD})$ produced by a new and old probe using 'standard settings' (2000 ms, $2000 \mathrm{~mW})$ on grey shades 6-9.

result of reflections at the glass/air interface. Although this test will provide an absolute value for the probe output, this type of assessment is not practical in the clinical setting and therefore a more practical method is required.

The method described in this paper is simple to perform, does not demand special calculations to be performed, or require special skills by the operator. The reproducibility is indicated by consistency of the results with different operators, different batches of grey scale sheets, and different batches of new probes. The data also show that this testing system can reliably detect changes in probe output of $20 \%$ or more when the laser is set above $1500 \mathrm{~mW}$.

The G-probe is designed to create a burn near the probe tip when applied to the conjunctiva and sclera, 
whereas in the testing system the probe is in contact with air where the emitted light will then diverge from the probe tip and then be absorbed on the grey scale. Although there is a distinct difference between the testing system and that of clinical use, the results do show that increasing burn size is associated with increasing laser output. The correlation of increasing burn size to the output provides validity to this method despite the fact that the absolute output at the probe tip has not been measured. Thus, the method indicates, in a semiquantitative way, the G-probe output by its ability to produce a burn on a test sheet.

Surprisingly, the study also shows that the effectiveness of the laser, using our testing system, varies more with changes to the power setting than changes to the time setting when the total energy output is greater than $4 \mathrm{~J}$. A 'threshold' burn is more likely to occur at a higher power setting than higher time setting, even when the total energy delivered is the same. This discrepancy may be because of the low thermal conductivity paper, allowing less dissipation of heat in a short time period, with the resulting effect that high powers at short time periods are more likely to cause burns than vice versa.

The results also show that 'threshold' burns are more likely to be produced with higher grey densities indicating that the amount of visible ciliary body pigmentation may be significant in determining the degree of ciliary body ablation that is produced following cyclodiode therapy.

Additionally, the results show that a reused probe is less effective at $2000 \mathrm{~mW}$ and $2000 \mathrm{~ms}$ in producing threshold burn on the threshold grey scale of 8 than a new probe. As indicated in the case of a reused probe (six previous uses), the output of the probe is reduced. However, the results of this study also indicate that by adjusting the laser setting, 'threshold burns' may be produced by the probe in question.

Since we have found that a G-probe using diode laser settings of $2000 \mathrm{~mW}$ and $2000 \mathrm{~ms}$ produces burns (approximately $1700 \mu \mathrm{m}$ ) on grey scale 8 , we suggest that those clinicians reusing probes use this test prior to commencement of a cyclodiode therapy. The G-probe can be tested using the chart as shown in Figure $2 \mathrm{~d}$, the test burns can then be compared to the scale given in the legend on the chart (the chart includes a series of dots of different sizes and the $1700 \mu \mathrm{m}$ size is indicated by an arrow). When a burn on the grey scale has reached the standard size of burn produced for 'standard settings' (ie burn size of $1700 \mu \mathrm{m}$ or more), then the G-probe is 'calibrated' and is ready for use on the patient. Any change in threshold value can then be compensated by appropriate changes to the time and power settings so that a probe produces threshold burns on scale 8. Such adjustments may therefore be useful to 'compensate' for the loss of laser output experienced with repeated use of the probe. The system may also be used to test the efficacy of new probes to ensure that damage to the probe has not occurred in transit.

Although the effect of laser ciliary body ablation is dependent on many variable factors such as ciliary body pigmentation or scleral thickness, ${ }^{13}$ standardisation of the treatment protocol has been shown to lead to a pressure lowering effect with relatively few complications. ${ }^{6}$ Hence, the prior testing of the G-probe in hospitals where clinicians are reusing probes, is another step that may lead to a 'standard' response following therapy.

In summary, this study shows that the practice of reuse of G-probes by ophthalmologists who perform cyclodiode therapy is widespread. This study demonstrates a practical test that can be used by those clinicians who reuse probes. The simplicity of the method means that the test can be performed without sophisticated equipment and with ease in the clinical setting. This strategy for probe assessment may lead to a more reliable amount of ciliary body ablation and to a more predictable intraocular pressure reduction following treatment.

\section{References}

1 Gaasterland DE, Pollack IP. Initial experience with a new method of transcleral cyclophotocoagulation for ciliary ablation in severe glaucoma. Trans Am Ophthalmol Soc 1992; 90: 225-246.

2 Bloom PA, Tsai JC, Sharma K et al. 'Cyclodiode'. Transscleral diode laser cyclophotocoagulation in the treatment of advanced refractory glaucoma. Ophthalmology 1997; 104: 1508-1519.

3 Kosoko O, Gaasterland DE, Pollack IP, Enger CL. Long-term outcome of initial ciliary ablation with contact diode laser transcleral cyclophotocoagulation for severe glaucoma. The Diode Laser Ciliary Ablation Study Group. Ophthalmology 1996; 103: 1294-1302.

4 Weekers R, Lavergne G, Watillon M et al. Effects of photocoagulation of ciliary body upon ocular tension. Am J Ophthalmol 1961; 52: 16-163.

5 Bietti. Surgical interventions on the ciliary body. New trends for the relief of glaucoma. JAMA 1950; 889-897.

6 Spencer AF, Vernon SA. 'Cyclodiode': results of a standard protocol. Br J Ophthalmol 1999; 83: 311-316.

7 Fishbaugh J. Overview and new technology in cyclodestructive procedures. Insight 1994; 19: 26-29.

8 Hawkins TA, Stewart WC. One-year results of semiconductor transcleral cyclophotocoagulation in patients with glaucoma. Arch Ophthalmol 1993; 111: 488-491.

9 Hennis HL, Stewart WC. Semiconductor diode laser transcleral cyclophotocoagulation in patients with glaucoma. Am J Ophthalmol 1992; 113: 81-85. 
10 Iris Medical Systems. Oculite Instructions, 1999.

11 British Standards BS EN ISO14971: 2000. Medical devices - application of risk management to medical devices. British Standards Institute, London, 2000.

12 Bock CJ, Freedman SF, Buckley EG, Shields MB. Transcleral diode laser cyclophotocoagulation for refractory paediatric glaucomas. J Paediatr Ophthalmol Strabismus 1997; 34: 235-239.

13 Brancato R, Carassa RG, Bettin P, Fiori M, Trabucchi G. Contact transcleral cyclophotocoagulation with diode laser in refractory glaucoma. Eur J Ophthalmol 1995; 5: 32-39.
14 Palmer DJ, Cohen J, Torczynski E, Deutsch TA. Transcleral diode laser cyclophotocoagulation on autopsy eyes with abnormally thinned sclera. Ophthal Surg Lasers 1997; 28: 495-500.

15 Feldman RM, El-Harazi SM, LoRusso FJ, McCash C, Lloyd WC, Warner PA. Histopathologic findings following contact transcleral semiconductor diode laser cyclophotocoagulation in a human eye. J Glaucoma 1997; 6: 139-140.

16 Schuman JS, Jacobson JJ, Puliafito CA, Noecker RJ, Reidy WT. Experimental use of semiconductor diode laser in contact transcleral cyclophotocoagulation in rabbits. Arch Ophthalmol 1990; 108: 1152-1157. 